\title{
Simulation of 3D Porous Media Flows with Application to Polymer Electrolyte Fuel Cells
}

\author{
N. I. Prasianakis ${ }^{1, *}$, T. Rosén ${ }^{1,2, \dagger}$, J. Kang ${ }^{1}$, J. Eller ${ }^{2}$, J. Mantzaras ${ }^{1}$ and \\ F. N. Büchi ${ }^{2}$ \\ ${ }^{1}$ Combustion Research Laboratory, Paul Scherrer Institute, Villigen PSI 5232, \\ Switzerland. \\ 2 Electrochemistry Laboratory, Paul Scherrer Institute, Villigen PSI 5232, \\ Switzerland.
}

Received 31 October 2011; Accepted (in revised version) 31 January 2012

Available online 29 August 2012

\begin{abstract}
A 3D lattice Boltzmann (LB) model with twenty-seven discrete velocities is presented and used for the simulation of three-dimensional porous media flows. Its accuracy in combination with the half-way bounce back boundary condition is assessed. Characteristic properties of the gas diffusion layers that are used in polymer electrolyte fuel cells can be determined with this model. Simulation in samples that have been obtained via X-ray tomographic microscopy, allows to estimate the values of permeability and relative effective diffusivity. Furthermore, the computational LB results are compared with the results of other numerical tools, as well as with experimental values.
\end{abstract}

AMS subject classifications: 65D18, 76G25, 76M28, 76S05

Key words: Porous media, GDLs, lattice Boltzmann, fuel cells, fluid dynamics.

\section{Introduction}

The simulation of porous media flows has recently attracted a lot of attention. The engineering applications related to porous media flows, are of interest in many industrial sectors that span from the chemical and oil industry to the fuel cell development. Polymer electrolyte fuel cells (PEFCs) have been considered in the last decades as a promising solution for future mobile and stationary energy conversion systems. Although the overall fuel cell performance has been substantially improved the last years, a significant

\footnotetext{
*Corresponding author. Email addresses: nikolaos.prasianakis@psi.ch (N. I. Prasianakis), rosen@ mech.kth.se (T. Rosén), jinfen.kang@psi.ch (J. Kang), jens.eller@psi.ch (J. Eller), ioannis.mantzaras@ psi.ch (J. Mantzaras), felix.buechi@psi.ch (F. N. Büchi)

${ }^{\dagger}$ Current address: Linné FLOW Centre, KTH Mechanics, SE-100 44 Stockholm, Sweden.
} 
limitation still persists related to the mass transport losses in the so-called gas diffusion layers (GDLs). The GDL's main purpose is to collect current over the flow channels and to provide uniform access of the fuel and oxidant gases to the electrocatalyst. This requires not only high permeability and relative diffusivity in the pore space, but also high conductivity in the solid. Permeability and diffusivity in the pore space are crucial to PEFC performance, as they determine the flux of reactants to the MEA (membrane-electrodeassembly). Testing various designs in a computer model using flow solvers is simpler and more efficient than carrying out the actual experiments. The GDL has a porosity of around $80 \%$ and is typically composed of carbon fibers with diameters typically of the order of 6-10 $\mathrm{mm}$. The pore size distribution shows a maximum in the range of 20$40 \mu m$ [1]. The internal structure of the GDL and the nature of the surfaces can play a significant role for the transport of gases inside the GDL. The transport properties of the material can change due to compression [2], or due to the presence of water under normal operating conditions of the fuel cell [3]. The use of computational methods along with structures obtained via X-ray tomographic microscopy (XTM), can provide the insight needed to optimize the design and the working efficiency of such devices [4-7]. Using a flow solver, apart from optimizing the flux of reactants to the MEA, it can also determine if water accumulation can be correlated to high/low velocity regions of the GDL (or other flow properties). As a result, regions where water accumulates can be reduced by optimizing the design.

Lately, the lattice Boltzmann method has received a lot of attention due to its strong kinetic-based theoretical background and its computational efficiency [8]. A domain of application of the method, usually considered as its strong asset, is the flows in porous media. For three dimensional complex geometry simulations, there exists a multitude of candidate lattice Boltzmann models. The usual 3D standard lattices are extensions of the two-dimensional with nine discrete velocities, the D2Q9, in three dimensions. Depending on the number of discrete velocities there exist: the D3Q13, the D3Q15, the D3Q19 and the D3Q27 [8-11] models. One of the characteristic features of these lattices is that communication and exchange of information occurs only within the next neighboring nodes. This results in: a) a simple implementation of complex geometry boundary conditions, and b) efficient parallelization of the algorithm. For the aforementioned lattices, the larger the number of discrete velocities, the better the accuracy and the correspondence to the continuous kinetic theory. At this point, accuracy refers to the ability of recovering the correct value of the relevant equilibrium higher order moments needed for establishing the Navier-Stokes at the continuum limit. It should be noted that this is not strictly true for extended lattices (beyond next-neighbor communication) and specific rules need to be applied in order to guarantee increase in accuracy when constructing such lattices [12-16]. All aforementioned next-neighbor lattices are lacking Galilean invariance, rotational isotropy, and reference temperature independence [12,17]. For small magnitude of velocity, (Mach number $\ll 1$ ), these deficiencies are negligible, while for larger values they become more pronounced, manifesting unphysical behavior. Among these models, the most accurate velocity set for isothermal flows, is the D3Q27 guided equi- 
librium model, which retains only a cubic error in some of its third order moments [17]. Similar to its D2Q9 equivalent, with a small added computational cost through the addition of appropriate correction terms, all deficiencies can be removed [18]. The same lattice is also the most appropriate candidate for simulation of thermal flows on standard lattices, again through the addition of appropriate correction terms [17].

Characterizing the computational lattice nodes as solid or void is straightforward, and the rules that the populations must obey on the boundaries can be quite simple. As a first approximation, the bounce-back boundary condition has been used. Several studies pointed out uncontrolled and unphysical velocity slip on the boundary, especially when the computational domain is discretized with a small number of grid points. This leads to a slightly improved boundary condition, which results to the so called 'half-way' bounceback (HBB) boundary condition [19]. For a specific value of the relaxation parameter $\tau$, and even for a small number of grid points, the inaccurate velocity slip vanishes and the aforementioned formulation can successfully simulate a no-slip boundary condition. The fixed value of the relaxation parameter, restricts the method for accurate calculations of the flow through porous media to small Reynolds numbers (Re), i.e., falling in the Darcylaw regime $(\operatorname{Re} \ll 1)$. This restriction has been studied in detail, and many LB models and boundary conditions have been proposed to overcome this issue [20,21].

In this paper, a 3D LB approach is presented and used to simulate the flow through complex three-dimensional geometries. The model is described by twenty seven discrete velocities and the collision is a single relaxation BGK type process. The behavior of the model when the HBB boundary condition is used, is studied in detail and its discretization error is computed. A direct application of the model in calculating the permeability and the relative diffusivity of a specific GDL material is also presented. Simulation results are then compared to experimental results as well as to other numerical methods for validation purposes.

The paper is organized as follows: in Section 2, the D3Q27 lattice Boltzmann model and its forcing method is presented. In Section 3, the accuracy of the method in combination with the HBB boundary condition is assessed. In Sections 4 and 5, flow through GDL porous materials is studied in detail and values of permeability and relative effective diffusivity are calculated.

\section{Lattice Boltzmann model: D3Q27 guided equilibrium}

For the simulations conducted in this paper, the guided equilibrium model is used [17]. Starting point is the definition of the velocity set. The lattice considered is the D3Q27 as illustrated in Fig. 1. The first nine discrete velocities $c_{i}$ are chosen to be the same as in the D2Q9 model. The discrete velocities for $i=0-8$ are defined as:

$$
\begin{aligned}
& c_{i x}=\{0,1,0,-1,0,1,-1,-1,1\}, \\
& c_{i y}=\{0,0,1,0,-1,1,1,-1,-1\}, \\
& c_{i z}=\{0,0,0,0,0,0,0,0,0\} .
\end{aligned}
$$




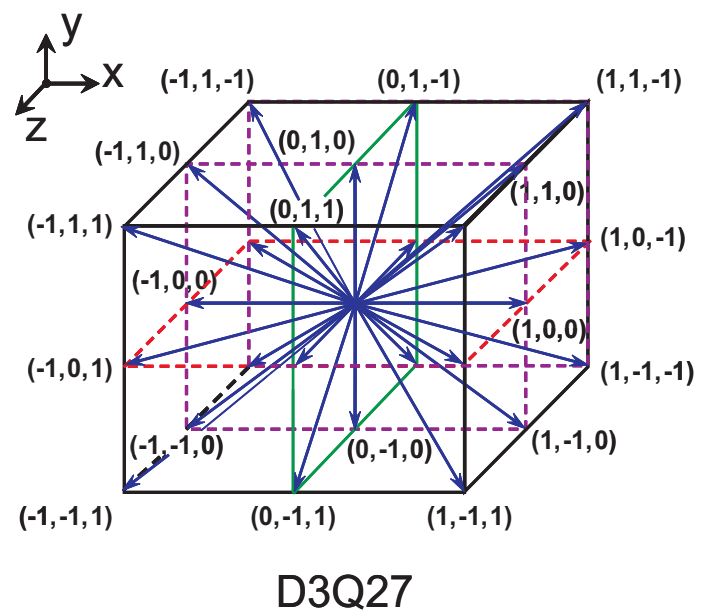

Figure 1: Three dimensional 27-velocity lattice (D3Q27). Every node on the cubic lattice corresponds to a different discrete population. Only next-neighbor communication is required for this space-filling lattice.

The next set of discrete velocities $i=9-17$ are the ones with positive $z-$ direction values $(x, y,+1)$ and they are defined as:

$$
\begin{aligned}
& c_{i x}=\{0,1,0,-1,0,1,-1,-1,1\}, \\
& c_{i y}=\{0,0,1,0,-1,1,1,-1,-1\}, \\
& c_{i z}=\{1,1,1,1,1,1,1,1,1\} .
\end{aligned}
$$

Finally, the set of discrete velocities $i=18-26$ are the ones with the property of negative $z$ - direction values $(x, y,-1)$ and they are defined as:

$$
\begin{aligned}
& c_{i x}=\{0,1,0,-1,0,1,-1,-1,1\}, \\
& c_{i y}=\{0,0,1,0,-1,1,1,-1,-1\}, \\
& c_{i z}=\{-1,-1,-1,-1,-1,-1,-1,-1,-1\} .
\end{aligned}
$$

For the D3Q27 model, the entropy function $H$, is chosen to have the Boltzmann-type form, with $f_{i}$ being the discrete velocity populations:

$$
H=\sum_{i=0}^{26} f_{i} \ln \left(f_{i}\right) .
$$

The local equilibrium constraints for the three dimensional D3Q27 model are defined with the help of the discrete velocity populations, at equilibrium $f_{i}^{\text {eq }}$ :

$$
\sum_{i=0}^{26} f_{i}^{e q}=\rho, \quad \sum_{i=0}^{26} c_{i \alpha} f_{i}^{e q}=j_{\alpha}, \quad \sum_{i=0}^{26} c_{i}^{2} f_{i}^{e q}=3 \rho T+\frac{j^{2}}{\rho} .
$$

where $\rho, j_{\alpha}=\rho u_{\alpha}$ and $T$ are the density, momentum and temperature fields respectively. 
The equilibrium populations for the D3Q27 basis can be derived as a minimizer of the entropy function (2.4) under specific constraints provided by local conservation laws $[16,22,23]$. Accuracy can be further increased when the condition, which stipulates that the equilibrium pressure tensor $P_{\alpha \beta}^{e q}$ is equal to the expression predicted by the kinetic theory, is added $[17,24]$. The guided equilibrium populations contain velocity terms up to the sixth order $\left(u^{6}\right)$ and can be written as:

$$
f_{i}^{e q}=\rho \prod_{a=x, y, z} \frac{\left(2 c_{i a}^{2}-1\right)}{2^{c_{i a}^{2}}}\left(c_{i a}^{2}-1+c_{i a} u_{a}+u_{a}^{2}+T\right) .
$$

It is clarified that at the reference temperature $T_{0}=1 / 3$, deviation from the NavierStokes-Fourier hydrodynamic equations is minimum $[23,24]$. Note that for the zerovelocity case, where $u=0$, the equilibrium population positivity constraint implies the temperature constraint $T \in] 0,1]$.

The simple form and the repeated patterns that generate the equilibrium populations, allow the fast evaluation of equilibrium values. The higher order moments of these populations deviate from the Maxwell Boltzmann moments similarly to the D2Q9 guided equilibrium thermal model. With the addition of appropriate correction terms at the level of the lattice-BGK equation, a Galilean invariant, rotational isotropic and reference temperature independent model is developed. It can then be used to study thermal flows in porous media, as well as acoustic problems. In the following, the guided equilibrium thermal model is operated at isothermal conditions, namely at the reference temperature $T_{0}=1 / 3$. For that, in the population expressions the temperature $T$, is replaced with $T_{0}$.

The implementation of the external forcing field follows the path presented in $[24,25]$. Forcing terms $\Psi_{i}$ are added to the Boltzmann BGK equation

$$
\partial_{t} f_{i}+c_{i \alpha} \partial_{\alpha} f_{i}=-\frac{1}{\tau}\left(f_{i}-f_{i}^{e q}\right)+\Psi_{i}
$$

with $\tau$ being the relaxation time. These terms act solely in the momentum equation, and can be used to introduce the counter terms of [18], which results in a Galilean invariant scheme. Since in all the simulations that follow, the magnitude of bulk velocity is small (Darcy law regime), correction terms can be switched off.

Forcing-terms are acting with specific weights with respect to the population and the space direction. The weight vectors $\Psi_{x}^{*}, \Psi_{y}^{*}, \Psi_{z}^{*}$ are introduced. For populations $i=0-8$ :

$$
\begin{aligned}
& \Psi_{x, i=0-8}^{*}=\left\{0, \frac{3}{2}, 0,-\frac{3}{2}, 0,-\frac{3}{8}, \frac{3}{8}, \frac{3}{8},-\frac{3}{8}\right\}, \\
& \Psi_{y, i=0-8}^{*}=\left\{0,0, \frac{3}{2}, 0,-\frac{3}{2},-\frac{3}{8},-\frac{3}{8}, \frac{3}{8}, \frac{3}{8}\right\}, \\
& \Psi_{z, i=0-8}^{*}=\{0,0,0,0,0,0,0,0,0\} .
\end{aligned}
$$


For populations $i=9-17$ :

$$
\begin{aligned}
& \Psi_{x, i=9-17}^{*}=\left\{0,-\frac{3}{8}, 0, \frac{3}{8}, 0, \frac{1}{8},-\frac{1}{8},-\frac{1}{8}, \frac{1}{8}\right\}, \\
& \Psi_{y, i=9-17}^{*}=\left\{0,0,-\frac{3}{8}, 0, \frac{3}{8}, \frac{1}{8}, \frac{1}{8},-\frac{1}{8},-\frac{1}{8}\right\}, \\
& \Psi_{z, i=9-17}^{*}=\left\{\frac{3}{2},-\frac{3}{8},-\frac{3}{8},-\frac{3}{8},-\frac{3}{8}, \frac{1}{8}, \frac{1}{8}, \frac{1}{8}, \frac{1}{8}\right\} .
\end{aligned}
$$

Finally for populations $i=18-26$ :

$$
\begin{aligned}
& \Psi_{x, i=18-26}^{*}=\left\{0,-\frac{3}{8}, 0, \frac{3}{8}, 0, \frac{1}{8},-\frac{1}{8},-\frac{1}{8}, \frac{1}{8}\right\}, \\
& \Psi_{y, i=18-26}^{*}=\left\{0,0,-\frac{3}{8}, 0, \frac{3}{8}, \frac{1}{8}, \frac{1}{8},-\frac{1}{8},-\frac{1}{8}\right\}, \\
& \Psi_{z, i=18-26}^{*}=\left\{-\frac{3}{2}, \frac{3}{8}, \frac{3}{8}, \frac{3}{8}, \frac{3}{8},-\frac{1}{8},-\frac{1}{8},-\frac{1}{8},-\frac{1}{8}\right\} .
\end{aligned}
$$

Finally, the forcing field $\mathbf{F}_{\alpha}$ enters the lattice BGK equation as:

$$
\Psi_{i}=\Psi_{i \alpha}^{*} \mathbf{F}_{\alpha}
$$

Following [18, 24], the lattice Bhatnagar-Gross-Krook (BGK) equation with a transformation similar to the one introduced in [26] reads:

$$
\begin{aligned}
& g=f+\frac{\delta t}{2 \tau}\left(f-f^{e q}\right)-\frac{\delta t}{2} \Psi \\
& g_{t+\delta t}=g_{t}+\frac{2 \delta t}{\delta t+2 \tau}\left[f_{t}^{e q}-g_{t}\right]+\frac{2 \tau \delta t}{\delta t+2 \tau} \Psi
\end{aligned}
$$

where the time step is set $\delta t=1$. Via Chapman-Enskog analysis it can be shown that the model recovers the isothermal Navier-Stokes equations with an error of the order $\left(u^{3}\right)$, and the dynamic viscosity is related to the relaxation parameter $\tau$ as, visc $=\tau \rho T_{0}$. Computation is carried out on the level of the transformed populations $g$. The equilibrium populations $f^{e q}$, needed in Eq. (2.13), are computed with the help of the moments of Eq. (2.12), which relate the locally conserved moments of the populations $f$ with the moments of the transformed populations $g$. Thus, the density $\rho(f)$ and the velocity $\mathbf{u}$ are evaluated as follows:

$$
\rho(f)=\rho(g), \quad \mathbf{u}(f)=\mathbf{u}(g)-\frac{\delta t}{2 \rho} \mathbf{F}
$$




\section{Accuracy dependence on the numerical viscosity: plane Poiseuille flow}

The accuracy of the current forcing implementation has been assessed following an analysis similar to the one presented in [19]. Exact analytic solutions of the flow field, for the case of the Poiseuille flow between two parallel plates ( $x y$-planes) have been derived. The half-way bounce-back boundary condition is used. In Fig. 2, the accuracy of the algorithm is presented for the forced-driven Poiseuille flow. On the vertical axis, the error refers to the difference of the obtained velocity profile (LB-method) from the continuum solution of the Poiseuille flow under no-slip boundary condition (Navier-Stokes). On the horizontal axis, the values of the viscosity in lattice Boltzmann units are depicted. Different curves correspond to channels of different size. $H$ is the number of computational nodes used to discretize the channel along the $z$-direction, and is equal to the distance between the two parallel planes. Simulation results are in excellent agreement and verify the theoretical analysis.

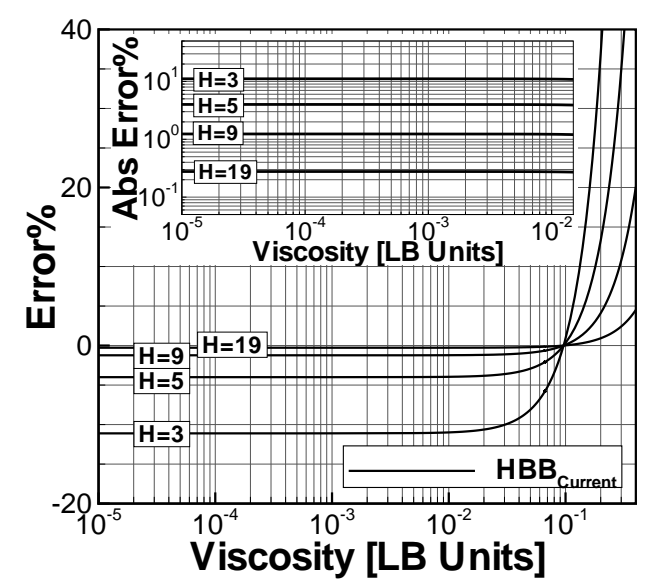

Figure 2: Difference in \% from the exact Navier-Stokes no-slip solution of the 3D Poiseuille flow between two parallel plates. Error $\%=\left(\left(U_{L B}-U_{N S}\right) / U_{N S}\right) \cdot 100$. $H$ refers to the number of computational nodes used for the discretization of the channel.

From Fig. 2, very useful information can be extracted. Similar to the standard D2Q9 LB formulation, as well as to other 3D LB implementations, there exists an optimum value of viscosity for which the LB solution is matching, to machine precision, with the NavierStokes solution, regardless of the number of nodes used for discretization. This behavior is typical for LB models combined with the HBB BC. For the D3Q27 model with the forcing procedure as presented in this paper, the value of the optimum viscosity is:

$$
\text { visc }_{o p t, D 3 Q 27}=\frac{1}{6 \sqrt{3}} \text {. }
$$

For smaller values, and for a large range of the numerical viscosity (visc $<$ visc $\left.\mathcal{o p t}_{\text {, }} \mathrm{DQ} Q 27\right)$, the error remains almost constant and decreases when the number of nodes $H$ increases, 
following second order spatial convergence. For a channel of $H=5$, the error is $4 \%$ and for a channel of $H=20$, the error is $0.2 \%$. For values of the viscosity larger than $v i s c_{o p t, D 3 Q 27}$, the error increases rapidly.

It is useful at this point to regard the current results under the prism of the Knudsen number. The Knudsen number can be defined as $K n=\sqrt{3} v i s c / H$, and is directly proportional to the value of the viscosity in lattice Boltzmann units. Therefore, high values of the viscosity correspond to high values of the $K n$ number. Also, the difference from the Navier-Stokes solution can be regarded as the slip velocity that originates from the kinetic nature of the lattice Boltzmann method. In that sense, the rightmost part of the diagram $\left(\right.$ visc $>$ visc $\left.{ }_{\text {opt }, \mathrm{D} 3 \mathrm{Q}_{27}}\right)$ predicts qualitatively that slip effects are more pronounced as soon as $K n$ is increased. Of course the HBB boundary condition fails to give quantitatively correct results. For better microflow description at non-vanishing Knudsen numbers, the diffusive boundary condition must be used $[20,27]$. Several boundary conditions have been developed in an effort to improve the accuracy $[28,29]$. For the present study, the HBB boundary condition is used, due to its simplicity and the fact that the solid boundaries of the porous media are already in a "staircase" form due to the pixel resolution of the X-ray tomographic images.

\section{Characterization of gas diffusion layers properties: permeability and relative effective diffusivity}

The efficiency of polymer electrolyte fuel cell systems (PEFCs) is highly dependent on the flow properties of the porous materials through which the fuel and oxidizer are transported. The components whose properties need to be examined are the so-called gas diffusion layers (GDLs). With the help of fluid dynamics algorithms, the transport properties of these materials can be measured (e.g. permeability and diffusivity). In order to assemble the fuel cell to the final stack product, the GDL is compressed and its size and geometry change. By doing so, the values of permeability and diffusivity are altered [2]. Moreover, the properties can change due to the presence of liquid water (produced from condensation of water vapor) in the porous structure, which blocks some channels and thus reduces the effective void area of the GDL under real operating conditions.

Permeability can be regarded as the quantity that describes the resistance of the material to the flow. According to the Darcy law, the convective transport of a viscous fluid through a porous medium, for small values of the Reynolds number $(\operatorname{Re} \ll 1)$, is described as:

$$
\vec{u}_{\text {mean }}=-\frac{k}{\rho v} \nabla p
$$

with $\vec{u}_{\text {mean }}$ being the volumetric mean velocity of the fluid in the domain under consideration, $k$ the permeability matrix, $v$ the kinematic viscosity and $\nabla p$ the applied pressure gradient. For the simulations that follow, instead of a pressure gradient $\nabla p$, an equiva- 
lent force field has been applied such that the resulting density gradient becomes negligible. Moreover, driving the flow via a forcing field allows the implementation of periodic boundary conditions, which in turn leads to the simulation of infinitely repeated sample geometry. This eliminates inflow/outflow boundary condition difficulties. For the permeability simulations of the next section, the flow is driven by a constant external forcing field. To measure the permeability of the specimen in the $x$-direction, $\mathbf{F}_{x}=$ const, $\mathbf{F}_{y}=0$, $\mathbf{F}_{z}=0$ is set. It is observed that for the kind of flows presented in this paper, in the range of values of $\mathbf{F} \in\left[10^{-12}, 10^{-3}\right]$, the resulting steady state flow is characterized by Reynolds number $\operatorname{Re} \ll 1$ (Darcy-law regime) and the measured value of permeability remains constant. For the simulations that follow, $\mathbf{F}=10^{-5}$ in lattice units is set. Periodic boundary conditions have been applied in all directions.

Another important material property is the relative effective diffusivity. The diffusive transport is described through Fick's first law:

$$
J=-D_{a b}^{e f f} \nabla C, \quad D_{a b}^{e f f}=\frac{\varepsilon}{T r} D_{a b},
$$

with $J$ being the molar flux, $D_{a b}^{e f f}$ the effective diffusion matrix, $\nabla C$ the concentration gradient and $D_{a b}$ the bulk diffusivity matrix. The effective diffusivity can be computed, once the ratio $\varepsilon / T r$ is calculated. It is the ratio of the porosity $\varepsilon$ over the tortuosity $T r$. Tortuosity is a measure of the morphology of the porous space and is strictly defined only in two dimensional space. Since porosity is already known, the tortuosity is the quantity of interest to be measured. For the three dimensional space, the definition of tortuosity is not straightforward. In [30] the authors describe the tortuosity in a given direction $x$, by regarding planes normal to this direction. If the perpendicular distance from a starting plane $(x=0)$ to an arbitrary parallel plane in the porous media $(x=i)$ is defined by $L_{E}$, and the geodesic distance $L_{G}$ is the mean nearest distance in pore space between points on the planes, the tortuosity can be defined as $\operatorname{Tr}=L_{G} / L_{E}$. A first approximation in calculating the approximate geodesic distance $L_{G}$, is to use the streamlines of the resulting velocity vector field from the lattice Boltzmann flow simulations. This method will be used in the next section.

\section{Accuracy of the algorithm for porous media flows: application to GDLs}

In this section, the model behavior for 3D complex geometries is studied. For the current simulations, the GDL sample of Fig. 3 is used. The sample is from a Toray TGP-H060 carbon fiber paper. The voxel size is $1.85 \mu \mathrm{m}$, the structure was obtained from an XTM scan at the TOMCAT beamline at the Swiss light source (SLS) at Paul Scherrer Institute, PSI.

When the lattice Boltzmann method is combined with the half-way bounce-back boundary condition, the calculated values of permeability depend on the numerical viscosity. This dependence can be explained with the help of Fig. 2, which refers to the plane 


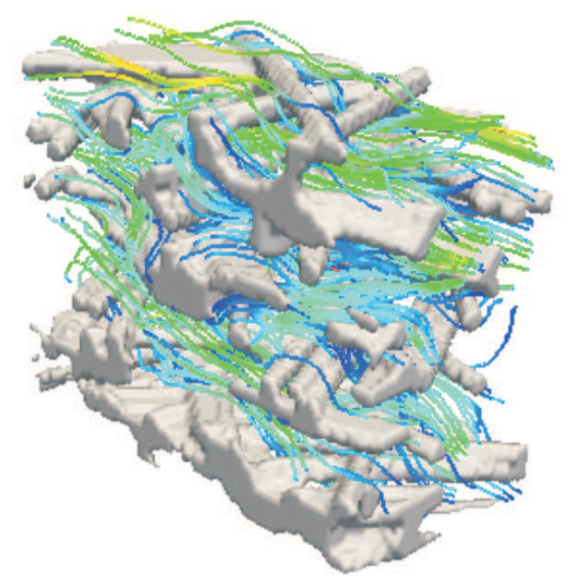

Figure 3: Streamlines in a sample of the gas diffusion layer. Solid structure is the gray part and the color of the streamlines represent the magnitude of fluid velocity at a given point. X-ray images of the TGP-H060 carbon fiber paper, were obtained from Swiss Light Source (SLS), PSI, at a resolution of $1.85 \mu \mathrm{m}$. Permeability and tortuosity can be directly extracted from the velocity vector field.

Poiseuille flow. The standard algorithm fails to describe accurately the flow in channels discretized with a small number of computational nodes. Consequently, the permeability value of a low resolution simulation will be accompanied with an error that originates exactly from these channels. There are mainly two strategies that can be followed in order to minimize the error introduced by the HBB boundary condition. Firstly, the numerical viscosity of the simulation can be set equal to the optimum viscosity value (visc opt,$D 3 Q 27)$. This in turn adds a restriction to the range of Reynolds numbers that can be simulated. High Reynolds number flows will require massive amount of grid points. Nevertheless, in porous media flows, where the interest is mostly in the Darcy-law regime $(\operatorname{Re} \ll 1)$, satisfactory results can be obtained. Secondly, especially when the flow is characterized by a larger Reynolds number $(R e>1)$, the smallest ducts in the porous medium must be discretized with an adequate number of grid points along with a small numerical viscosity value. This is achieved either by using finer grids in the entire computational domain, or via local computational grid refinement for a given geometry [31,32], at the region of the smallest channels.

In Fig. 4 the permeability values of the GDL sample of Fig. 3, obtained for different values of the numerical viscosity are plotted. For the current simulations, different resolutions of the same sample have been used. The dimensions of the sample obtained from the X-ray tomography via segmentation is $100 \times 50 \times 90$ pixels. Simulations after refining the computational grid have been also conducted and are presented in Fig. 4 . The refined grid has a size of 200x100x180 nodes. The calculated permeability values, as expected, exhibit a behavior similar to the error behavior of the Poiseuille flow. The best prediction of the algorithm is considered to be the result of the simulation at optimum viscosity $\left(v i s c_{o p t, D 3 Q 27}\right)$. For the finer grid, the number of channels that are discretized 


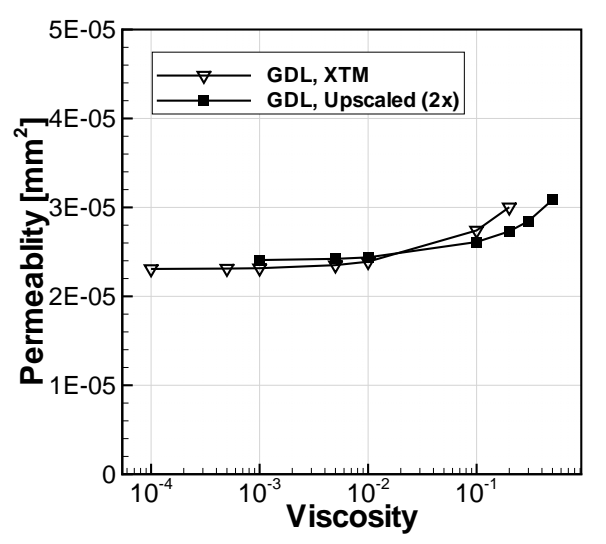

Figure 4: Permeability dependence on the numerical viscosity for a 3D GDL sample. Similar to the Poiseuille flow, for a large range of small values of viscosity the permeability does not change.

with a small number of nodes is reduced, and the simulation result becomes less sensitive to the change of the numerical viscosity.

Recently, Becker et al. [2], studied the influence of GDL compression on material properties by combining X-ray tomography and numerical simulations. XTM tomograms of Toray TGP-H060 carbon fiber paper were used at three different compression ratios: uncompressed, $20 \%$ compression and $40 \%$ compression. The voxel size is now $0.74 \mu \mathrm{m}$. Due to compression, the porosity and the overall geometrical properties of the sample change. The uncompressed sample consisted of $600 \times 600 \times 224$ voxels. Permeability values were calculated using the FFF-Stokes solver [33]. Also, the effective relative diffusivity was obtained by simulating diffusive transport using the explicit jump solver of [34]. In this paper, the same segmented structures have been used in order to compare the accuracy of the LB algorithm to the aforementioned numerical tools and to experimental results. The resolution of the tomographic images was used without any further computational grid refinement (native resolution). More information regarding the experimental methodologies for the determination of permeability and relative effective diffusivity, can be found in [2]. The results are presented in Figs. 5 and 6. Through-plane is the plane perpendicular to the reactant gas flows in the supply channels cutting through the entire MEA (membrane-electrode-assembly), while in plane refers to the plane parallel to the channel flows (see [2]). The permeability values, calculated via the LB simulations are in very good agreement with the results from the FFF-Stokes solver. Both numerical tools are in good agreement with the experimental results as well. The Stokes solver was more efficient in terms of computational time. The advantage of the LB algorithm is that it solves the full Navier-Stokes equations and thus can be used also for studying the flow in regimes where the flow is: a) laminar and non-linear, where the inertial forces start to become more important $(\operatorname{Re} \geq 1)$, and $b)$ non-laminar and non-linear $(\operatorname{Re} \gg 1)$. Moreover, the LB model presented in this paper can be used as a basis for the simulation of thermal flows in complex geometries. For the values of the effective relative diffusivity, however, 


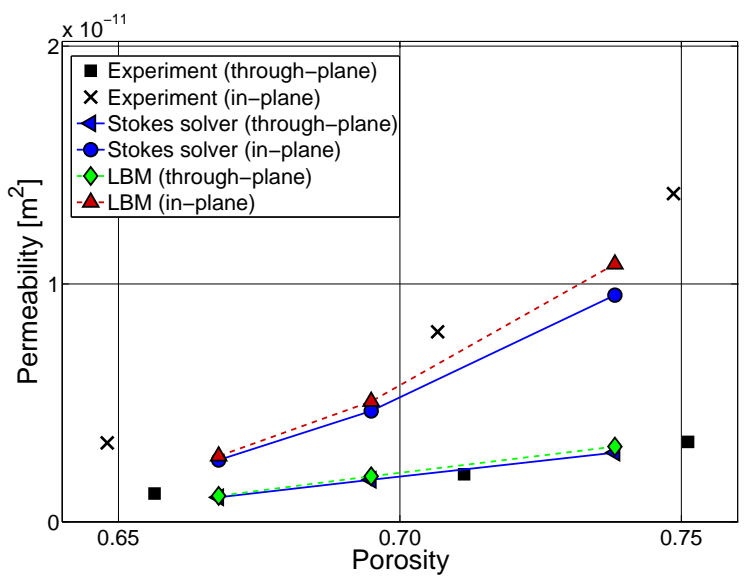

Figure 5: Permeability results for in-plane and through-plane cases, at different porosities, i.e. calculation for different compression rates $(0 \%, 20 \%$ and $40 \%)$. Experiments, Stokes-solver and LB results are compared.

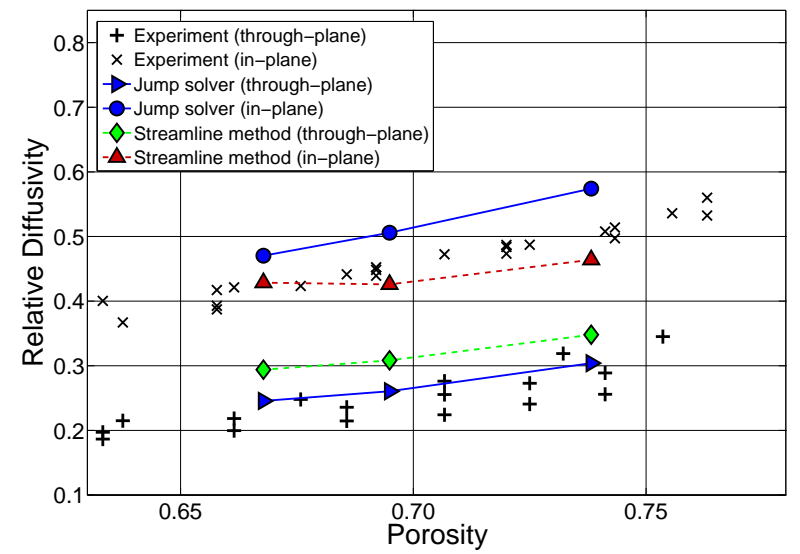

Figure 6: Relative effective diffusivity values, for in-plane and through-plane cases as function of porosity, i.e. for different compression rates $(0 \%, 20 \%$ and $40 \%)$. Experiments, jump-solver and streamline method based on the LB-results are compared.

significant quantitative difference exists between the LB-based calculation and the jump solver. The LB-based calculation yields better results for the in-plane case (600 nodes resolution) than the through-plane case (224 nodes resolution).

\section{Conclusions}

In this paper, a three dimensional LB model with twenty-seven discrete velocities has been presented. Its accuracy in conjunction with the half-way bounce back boundary condition has been studied analytically in detail for the case of Poiseuille flow. The accu- 
racy for complex geometry flows has also been assessed. For validation purposes, simulation results have been compared with different numerical tools (Stokes-solver, jump solver) as well as with experimental results. Values of permeabilities and relative effective diffusivities have been calculated for a Toray carbon fiber paper that is used as GDL material in polymer electrolyte fuel cells.

The LB algorithm presented in this paper, is planned to be used to also study the change of gas transport properties, due to the existence of liquid water in GDLs. This will be done using in-situ X-ray tomographic pictures obtained from the SLS facility of Paul Scherrer Institute, where water distribution can be distinguished from the GDL material. Simulations of such cases can provide important insight for the fuel cell research and development, since currently it is very difficult to experimentally extract this kind of information [3].

\section{Acknowledgments}

The authors would like to thank J. Becker for providing the exact numerical results and other relevant information of [2]. The authors would like to thank S. Succi and F. Lundell for useful discussions. Financial support from BFE via projects 103078 and 153708 is kindly acknowledged. T.R. acknowledges partial support from KTH Mechanics.

\section{Appendix: Guided equilibrium populations}

The guided equilibrium populations $f_{i}^{e q}$, have a computationally attractive form. For $i=0-8$ :

$$
\begin{aligned}
& f_{0}^{e q}=-\rho\left(-1+T+u_{x}^{2}\right)\left(-1+T+u_{y}^{2}\right)\left(-1+T+u_{z}^{2}\right), \\
& f_{1}^{e q}=(1 / 2) \rho\left(T+u_{x}+u_{x}^{2}\right)\left(-1+T+u_{y}^{2}\right)\left(-1+T+u_{z}^{2}\right), \\
& f_{2}^{e q}=(1 / 2) \rho\left(-1+T+u_{x}^{2}\right)\left(T+u_{y}+u_{y}^{2}\right)\left(-1+T+u_{z}^{2}\right), \\
& f_{3}^{e q}=(1 / 2) \rho\left(T-u_{x}+u_{x}^{2}\right)\left(-1+T+u_{y}^{2}\right)\left(-1+T+u_{z}^{2}\right), \\
& f_{4}^{e q}=(1 / 2) \rho\left(-1+T+u_{x}^{2}\right)\left(T-u_{y}+u_{y}^{2}\right)\left(-1+T+u_{z}^{2}\right), \\
& f_{5}^{e q}=-(1 / 4) \rho\left(T+u_{x}+u_{x}^{2}\right)\left(T+u_{y}+u_{y}^{2}\right)\left(-1+T+u_{z}^{2}\right), \\
& f_{6}^{e q}=-(1 / 4) \rho\left(T-u_{x}+u_{x}^{2}\right)\left(T+u_{y}+u_{y}^{2}\right)\left(-1+T+u_{z}^{2}\right), \\
& f_{7}^{e q}=-(1 / 4) \rho\left(T-u_{x}+u_{x}^{2}\right)\left(T-u_{y}+u_{y}^{2}\right)\left(-1+T+u_{z}^{2}\right), \\
& f_{8}^{e q}=-(1 / 4) \rho\left(T+u_{x}+u_{x}^{2}\right)\left(T-u_{y}+u_{y}^{2}\right)\left(-1+T+u_{z}^{2}\right) .
\end{aligned}
$$


Populations $f_{i}^{e q}$, for $i=9-17$ :

$$
\begin{aligned}
& f_{9}^{e q}=(1 / 2) \rho\left(-1+T+u_{x}^{2}\right)\left(-1+T+u_{y}^{2}\right)\left(T+u_{z}+u_{z}^{2}\right), \\
& f_{10}^{e q}=-(1 / 4) \rho\left(T+u_{x}+u_{x}^{2}\right)\left(-1+T+u_{y}^{2}\right)\left(T+u_{z}+u_{z}^{2}\right), \\
& f_{11}^{e q}=-(1 / 4) \rho\left(-1+T+u_{x}^{2}\right)\left(T+u_{y}+u_{y}^{2}\right)\left(T+u_{z}+u_{z}^{2}\right), \\
& f_{12}^{e q}=-(1 / 4) \rho\left(T-u_{x}+u_{x}^{2}\right)\left(-1+T+u_{y}^{2}\right)\left(T+u_{z}+u_{z}^{2}\right), \\
& f_{13}^{e q}=-(1 / 4) \rho\left(-1+T+u_{x}^{2}\right)\left(T-u_{y}+u_{y}^{2}\right)\left(T+u_{z}+u_{z}^{2}\right), \\
& f_{14}^{e q}=(1 / 8) \rho\left(T+u_{x}+u_{x}^{2}\right)\left(T+u_{y}+u_{y}^{2}\right)\left(T+u_{z}+u_{z}^{2}\right), \\
& f_{15}^{e q}=(1 / 8) \rho\left(T-u_{x}+u_{x}^{2}\right)\left(T+u_{y}+u_{y}^{2}\right)\left(T+u_{z}+u_{z}^{2}\right), \\
& f_{16}^{e q}=(1 / 8) \rho\left(T-u_{x}+u_{x}^{2}\right)\left(T-u_{y}+u_{y}^{2}\right)\left(T+u_{z}+u_{z}^{2}\right), \\
& f_{17}^{e q}=(1 / 8) \rho\left(T+u_{x}+u_{x}^{2}\right)\left(T-u_{y}+u_{y}^{2}\right)\left(T+u_{z}+u_{z}^{2}\right) .
\end{aligned}
$$

Finally, populations $f_{i}^{e q}$, for $i=18-26$ :

$$
\begin{aligned}
& f_{18}^{e q}=(1 / 2) \rho\left(-1+T+u_{x}^{2}\right)\left(-1+T+u_{y}^{2}\right)\left(T-u_{z}+u_{z}^{2}\right), \\
& f_{19}^{e q}=-(1 / 4) \rho\left(T+u_{x}+u_{x}^{2}\right)\left(-1+T+u_{y}^{2}\right)\left(T-u_{z}+u_{z}^{2}\right), \\
& f_{20}^{e q}=-(1 / 4) \rho\left(-1+T+u_{x}^{2}\right)\left(T+u_{y}+u_{y}^{2}\right)\left(T-u_{z}+u_{z}^{2}\right), \\
& f_{21}^{e q}=-(1 / 4) \rho\left(T-u_{x}+u_{x}^{2}\right)\left(-1+T+u_{y}^{2}\right)\left(T-u_{z}+u_{z}^{2}\right), \\
& f_{22}^{e q}=-(1 / 4) \rho\left(-1+T+u_{x}^{2}\right)\left(T-u_{y}+u_{y}^{2}\right)\left(T-u_{z}+u_{z}^{2}\right), \\
& f_{23}^{e q}=(1 / 8) \rho\left(T+u_{x}+u_{x}^{2}\right)\left(T+u_{y}+u_{y}^{2}\right)\left(T-u_{z}+u_{z}^{2}\right), \\
& f_{24}^{e q}=(1 / 8) \rho\left(T-u_{x}+u_{x}^{2}\right)\left(T+u_{y}+u_{y}^{2}\right)\left(T-u_{z}+u_{z}^{2}\right), \\
& f_{25}^{e q}=(1 / 8) \rho\left(T-u_{x}+u_{x}^{2}\right)\left(T-u_{y}+u_{y}^{2}\right)\left(T-u_{z}+u_{z}^{2}\right), \\
& f_{26}^{e q}=(1 / 8) \rho\left(T+u_{x}+u_{x}^{2}\right)\left(T-u_{y}+u_{y}^{2}\right)\left(T-u_{z}+u_{z}^{2}\right) .
\end{aligned}
$$

\section{References}

[1] R. Flückiger, S. A. Freunberger, D. Kramer, A. Wokaun, G. G. Scherer and F. N. Büchi, Anisotropic, effective diffusivity of porous gas diffusion layer materials for PEFC, Electrochim. Acta, 54, 551 (2008). 
[2] J. Becker, R. Flückiger, M. Reum, F. Büchi, F. Marone and M. Stampanoni, Determination of material properties of gas diffusion layers: Experiments and simulations using phase contrast tomographic microscopy, J. Electrochem. Soc., 156, B1175-B1181 (2009).

[3] J. Eller, T. Rosén, F. Marone, M. Stampanoni, A. Wokaun and F. N. Büchi, Progress in in situ X-Ray tomographic microscopy of liquid water in gas diffusion layers of PEFC, J. Electrochem. Soc., 158, B963 (2011).

[4] H. Ostadi, P. Rama, Y. Liu, R. Chen, X. X. Zhang and K. Jiang, Influence of threshold variation on determining the properties of a polymer electrolyte fuel cell gas diffusion layer in X-ray nano-tomography, Chem. Eng. Sci., 65, 2213 (2010).

[5] P. Rama, Y. Liu, R. Chen, H. Ostadi, K. Jiang, X. Zhang, R. Fisher and M. Jeschke, An X-ray tomography based lattice Boltzmann simulation study on gas diffusion layers of polymer electrolyte fuel cells, J. Fuel Cell Sci. and Tech., 7, 031015 (2010).

[6] X. D. Niu, T. Munekata, S. A. Hyodo and K. Suga, An investigation of water-gas transport processes in the gas-diffusion-layer of a PEM fuel cell by a multiphase multiple-relaxationtime lattice Boltzmann model, J. Power Sources, 172, 542 (2007).

[7] T. Munekata, T. Inamuro and S. A. Hyodo, Gas transport properties in gas diffusion layers: A lattice Boltzmann study, Commun. Comput. Phys., 9, 1335 (2011).

[8] S. Succi, The Lattice Boltzmann Equation for Fluid Dynamics and Beyond, Oxford University Press, Oxford, 2001.

[9] Y. H. Qian, D. D'Humieres and P. Lallemand, Lattice BGK models for Navier-Stokes equation, Europhys. Lett., 17, 479 (1992).

[10] R. Benzi, S. Succi and M. Vergassola, The lattice Boltzmann equation: Theory and applications, Phys. Rep., 222, 3 (1992).

[11] F. Higuera, S. Succi and R. Benzi, Lattice gas dynamics with enhanced collisions, Europhys. Lett., 9, 345 (1989).

[12] I. Karlin and P. Asinari, Factorization symmetry in the lattice Boltzmann method, Physica A, 389, 1530-1548 (2010).

[13] S. S. Chikatamarla and I. V. Karlin, Lattices for the lattice Boltzmann method, Phys. Rev. E, 79, 046701 (2009).

[14] X. Shan, X.-F. Yuan and H. Chen, Kinetic theory representation of hydrodynamics: A way beyond the Navier-Stokes equation, J. Fluid Mech., 550, 413 (2006).

[15] P. C. Philippi, L. A. Hegele, L. O. E. dos Santos and R. Surmas, From the continuous to the lattice Boltzmann equation: The discretization problem and thermal models, Phys. Rev. E, 73, 056702 (2006).

[16] S. Ansumali, I. V. Karlin and H. C. Öttinger, Minimal entropic kinetic models for hydrodynamics, Europhys. Lett., 63, 798 (2003).

[17] N. Prasianakis, Ph.D. Thesis, Swiss Federal Institut of Technology (ETH), Zurich, 2008.

[18] N. I. Prasianakis, I. V. Karlin, J. Mantzaras and K. B. Boulouchos, Lattice Boltzmann method with restored Galilean invariance, Phys. Rev. E, 79, 066702 (2009).

[19] X. He, Q. Zou, L.-S. Luo and M. Dembo, Analytic solutions of simple flows and analysis of nonslip boundary conditions for the lattice Boltzmann BGK model, J. Stat. Phys., 87, 115 (1997).

[20] S. Ansumali and I. V. Karlin, Kinetic boundary condition for the lattice Boltzmann method, Phys. Rev. E, 66, 026311 (2002).

[21] C. Pan, L.-S. Luo and C. T. Miller, An evaluation of lattice Boltzmann schemes for porous medium flow simulation, Computers and Fluids, 35, 898 (2006).

[22] I. V. Karlin, A. Ferrante and H. C. Öttinger, Perfect entropy functions of the lattice Boltzmann 
method, Europhys. Lett., 47, 182 (1999).

[23] S. Ansumali and I. V. Karlin, Consistent lattice Boltzmann method, Phys. Rev. Lett., 95, 260605 (2005).

[24] N. I. Prasianakis and I. V. Karlin, Lattice Boltzmann method for thermal flow simulation on standard lattices, Phys. Rev. E, 76, 016702 (2007).

[25] N. I. Prasianakis and I. V. Karlin, Lattice Boltzmann method for simulation of compressible flows on standard lattices, Phys. Rev. E, 78, 016704 (2008).

[26] X. He, S. Chen and G. D. Doolen, A novel thermal model for the lattice Boltzmann method in incompressible limit, J. Comput. Phys., 146, 282 (1998).

[27] S. Ansumali, I. V. Karlin, S. Arcidiacono, A. Abbas and N. I. Prasianakis, Hydrodynamics beyond Navier-Stokes: Exact solution to the lattice Boltzmann hierarchy, Phys. Rev. Lett., 98, 124502 (2007).

[28] O. Malaspinas, B. Chopard and J. Latt, General regularized boundary condition for multispeed lattice Boltzmann models, Computers \& Fluids, 49, 29-35 (2011).

[29] M. Sbragaglia and S. Succi, Analytical calculation of slip flow in lattice Boltzmann models with kinetic boundary conditions, Phys. Fluids, 17, 093602 (2005).

[30] C. J. Gommes, Practical methods for measuring the tortuosity of porous materials from binary or gray-tone tomographic reconstructions, AIChE J., 47, 2000, (2009).

[31] O. Filippova and D. Hanel, Grid refinement for lattice-BGK models, J. Comp. Phys., 147, 219 (1998).

[32] O. Filippova, S. Succi, F. Mazzocco, C. Arrighetti, G. Bella and D. Hanel, Multiscale lattice Boltzmann schemes with turbulence modeling, J. Comp. Phys., 147, 219 (1998).

[33] A. Wiegmann, Computation of the permeability of porous materials from their microstructure by FFF-stokes, Fraunhofer ITWM, Tech. Rep., 129, (2007).

[34] A. Wiegmann and A. Zemitis, Ej-heat: A fast explicit jump harmonic averaging solver for the effective heat conductivity of composite materials, Fraunhofer ITWM, Tech. Rep., 94, (2006). 